\title{
Erst Flop, jetzt Top!
}

\section{Vor drei Jahren war die mechanische Thrombektomie bei Schlaganfall auf- grund von drei negativen Studien tot ge- sagt worden. Fünf neue Studien zeigen das Gegenteil.}

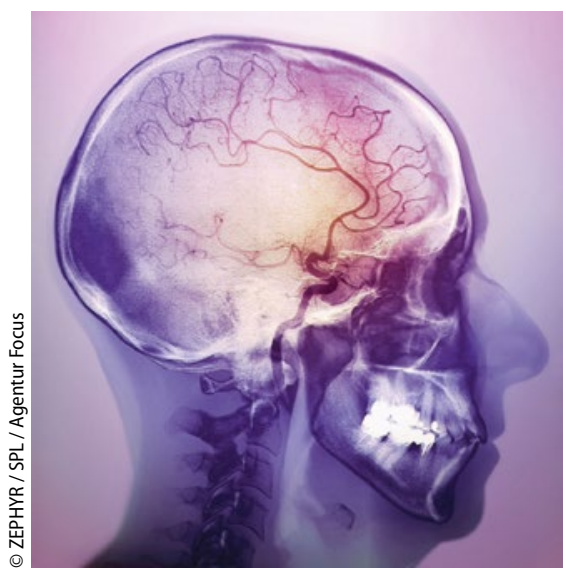

Eine Revolution nennt daher Prof. Dr. Hans-Christoph Diener, Direktor der Neurologischen Universitätsklinik Essen, jetzt die mechanische Thromboektomie: Laut einer aktuellen Metaanalyse liegt die Number needed to treat so günstig wie bei kaum einer anderen Maßnah- me [6]: Vier bis fünf Patienten müssen behandelt werden, damit einer profitiert.

Als Grund für die zunächst ungünstigen Studienausgänge nannte er zahlreiche methodische Mängel. Die neuen randomisierten Studien umfassten Patienten mit distalen Verschlüssen der Arteria carotis interna und der proximalen Arteria cerebri media, in denen eine systemische Thrombolyse im Zeitfenster von 4,5 Stunden mit einer systemischen Thrombolyse und zusätzlicher Thrombektomie mit einem Stent-Retriever untersucht wurde. „Im Gegensatz zu den Kardiologen hinterlassen wir keinen Müll“, meinte Diener: Der Stent wird nach dem Einfangen des Thrombus wieder entfernt.

Von fünf Studien wurden vier bereits vorzeitig beendet, weil schon bei Einschluss von einem Drittel der geplanten Patienten der primäre Endpunkt signifikant war: Wesentlich mehr Patienten erreichten einen Wert von 0 bis 1 auf der modifizierten Rankin-Skala, d.h. sie trugen keine oder kaum neurologische Ausfälle davon. „Die Wahrscheinlichkeit, dass Sie auf zwei Beinen das Krankenhaus verlassen, ist um 2,6 erhöht“, schwärmt Diener: „Das haben wir beim Schlaganfall noch nie erreicht.“

In den fünf Studien wurden insgesamt 633 Patienten mit Thrombolyse und Thrombektomie behandelt, und mit 650 Patienten mit systemischer Thrombolyse allein verglichen. Neben der 2,42fach erhöhten Wahrscheinlichkeit, den Schlaganfall nur mit minimalen neurologischen Ausfällen zu überleben, zeigte sich auch ein Trend zu einer verringerten Mortalität. Dabei kam es nicht zu mehr intrakraniellen Blutungen. Im Gegensatz zur systemischen Thrombolyse, bei der die Rekanalisationsraten zwischen 40 und 50\% liegen, wurden Raten von $70-90 \%$ erreicht.

"Jetzt brauchen wir mehr interventionelle Neuroradiologen“, fordert Diener, denn für ihn ist klar: An die Hirngefäße dürfen nur Neuroradiologen!

\section{Friederike Klein}

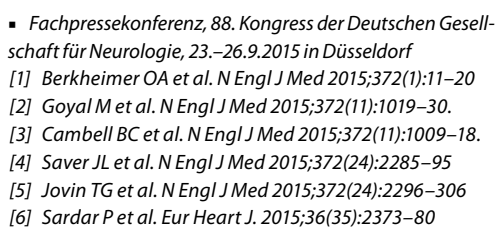

\section{Schlaganfallrisiko}

\section{Lebensbedingungen in der Kindheit relevant}

_ Herkömmliche Risikofaktoren erklären nicht vollständig, warum Schlaganfallinzidenz und -mortalität in Regionen mit niedrigem Einkommensniveau höher ist als in solchen mit hohem. Deshalb untersuchte die Fall-Kontroll-Studie GENESIS die Assoziation von sozioökomischen Parametern in Kindes-, Jugendund Erwachsenenalter bei 470 Patienten nach einem ischämischen Schlaganfall und 807 Kontrollen aus Ludwigshafen.

Wie zu erwarten waren die bekannten Schlaganfallrisikofaktoren wie Hypertonie, Diabetes, Hypercholesterinämie, Vorhofflimmern, PAVK, Rauchen, hoher Alkoholkonsum und fehlende körperliche Aktivität in der Gruppe der Schlaganfallpatienten häufiger als in der Kontrollgruppe. Korrigiert um diese Einflussfaktoren ergaben sich aber auch Hinweise auf den Einfluss sozioökonomischer Faktoren auf das Schlaganfallrisiko - und das bereits in der Kindheit, wie Prof. Dr. Armin Grau, Ludwigshafen, berichtete. So verdoppelte sich das Schlaganfallrisiko bei fehlender Berufsausbildung $(\mathrm{OR}=1,93$ als Faktor in der Adoleszenz bzw. OR = 1,99 als Faktor im Erwachsenenalter). Im Erwachsenenalter waren außerdem eine Arbeitslosigkeit über sechs Monate und der Familienstand (ledig, geschieden oder verwit- wet) mit einem erhöhten Schlaganfallrisiko assoziiert (OR = 1,52 bzw. 1,63).

Eine Assoziation zeigten auch ein später erhöhtes Schlaganfallrisiko mit dem Aufwachsen mit mehr als drei Geschwistern in der Kindheit (Odds Ratio [OR $]=1,48)$, dem Fehlen einer Toilette in der Wohnung (als Zeichen mangelhafter hygienischer Verhältnisse, in der Kindheit der vorwiegend älteren Studienteilnehmer noch relevant, OR 1,52) sowie ein niedriges Familieneinkommen in der Kindheit (OR 2,90).

- Mini-Symposium: Genetische, entzündliche und sozioökonomische Determinanten des ischämischen Schlaganfalls. 23.9.2015, DGN-Kongress in Düsseldorf 